\title{
Is Dual Linear Self-Calibration Artificially Ambiguous?
}

\author{
Pierre Gurdjos \\ ENSEEIHT - IRIT, Toulouse, France
}

\author{
Adrien Bartoli \\ CNRS - LASMEA, Clermont-Ferrand, France
}

\author{
Peter Sturm \\ LJK - INRIA, Grenoble, France
}

\begin{abstract}
This purely theoretical work investigates the problem of artificial singularities in camera self-calibration. Selfcalibration allows one to upgrade a projective reconstruction to metric and has a concise and well-understood formulation based on the Dual Absolute Quadric (DAQ), a rank3 quadric envelope satisfying (nonlinear) 'spectral constraints': it must be positive of rank 3. The practical scenario we consider is the one of square pixels, known principal point and varying unknown focal length, for which generic Critical Motion Sequences (CMS) have been thoroughly derived. The standard linear self-calibration algorithm uses the DAQ paradigm but ignores the spectral constraints. It thus has artificial CMSs, which have barely been studied so far.

We propose an algebraic model of singularities based on the confocal quadric theory. It allows to easily derive all types of CMSs. We first review the already known generic CMSs, for which any self-calibration algorithm fails. We then describe all CMSs for the standard linear selfcalibration algorithm; among those are artificial CMSs caused by the above spectral constraints being neglected. We then show how to detect CMSs. If this is the case it is actually possible to uniquely identify the correct selfcalibration solution, based on a notion of signature of quadrics. The main conclusion of this paper is that a posteriori enforcing the spectral constraints in linear self-calibration is discriminant enough to resolve all artificial CMSs.
\end{abstract}

\section{Introduction}

Structure-from-Motion (SfM) is the problem of recovering a metric model - the scene structure and the camera motion and intrinsic parameters- from multiple views. It has been extensively studied over the past few decades (see e.g. [5, 8]). The self-calibration paradigm allows one to solve SfM with few assumptions about the intrinsics and barely none about the structure and the motion, thus making $\mathrm{SfM}$ versatile and flexible. In theory, the mere zero-skew assumption is sufficient for the SfM problem to be wellposed [9]. We make the stronger standard assumption that the pixels are square and the principal point lies at the image centre, but that the focal length is time-varying.

One of the most remarkable results in SfM says that, given enough point correspondences, a projective reconstruction of $3 \mathrm{D}$ points and cameras can be computed $[6,4$, 7]. The metric upgrade of this model is a further reconstruction step, that draws on the equivalence of the projective to the metric model via an unknown 3D homography. This is mathematically formulated using the elegant geometrical paradigm of the Dual Absolute Quadric (DAQ) proposed by Triggs in 1997 [22], which has also been extended to that of the Absolute Quadratic Complex [13, 23]. The DAQ has nonlinear 'spectral constraints': it is semipositive and rank-3. This models the fact that the DAQ is the plane-envelope of the Absolute Conic, a virtual conic (i.e. consisting only of complex points). Once the DAQ is recovered, the upgrading homography is easily extracted from it. This class of algorithms has been popularised by the linear least-squares formulation of Pollefeys et al. [12, 17], which is hereafter dubbed dual linear selfcalibration. Recently, self-calibration approaches enforcing the spectral constraints while globally optimizing some algebraic error measure have been proposed [2, 3]. However, the above dual linear self-calibration approach is probably still the most used one.

As mentioned in [8, p498], 'self-calibration can work well in the right circumstances, but used recklessly it will fail', especially if one does not 'take care to avoid ambiguous motion sequences', so-called critical motion sequences (CMSS). For these, there exist more than one virtual conic satisfying the self-calibration constraints. Sturm [21] and Kahl et al. [10] studied this problem and established that there exist generic critical motion sequences for the camera, and that they defeat any self-calibration algorithm.

The SfM framework has all its basic building blocks in place. There is however an important missing piece regarding artificial CMSs. It has been known that there exist CMSs which are not generic for the self-calibration problem, but for which the dual linear self-calibration algorithm fails in finding the 'true' DAQ [20]. This is essentially due to this algorithm ignoring the spectral constraints. In other words, in generic criticality, only quadric envelopes 
representing a virtual conic can satisfy the self-calibration equations while, in artificial criticality, any quadric envelope (virtual or not, degenerate or not) can satisfy them. In the case where a linear self-calibration algorithm returns a 1D family of ambiguous solutions, previous works [12, 17] showed that the rank-deficiency constraint can be enforced a posteriori to select candidate solutions. Bartoli et al. [1] select the most realistic solution by examining the camera focal length.

In this paper, we propose a theoretical framework for exhaustively studying CMSs in dual linear self-calibration, based on the confocal quadric theory. Interestingly enough, this theory has been established long ago by famous mathematicians. The resulting framework can be viewed as an add-on to the DAQ model of self-calibration. The main conclusion of this paper is thus that for the studied selfcalibration problem, even linear algorithms only suffer from the generic CMSs, if spectral constraints are enforced a posteriori.

Paper organization. In section 2, the self-calibration problem and its ingredients are formulated and the notion of CMS is defined. Generic CMSs are reviewed in section 3. In section 4, we provide an exhaustive list of CMSs for dual linear self-calibration and highlight which of these are artificial, i.e. only exist due to neglecting the spectral constraints. Some details on the underlying theoretical analysis are provided in section 5 . In section 6 , we show how a projectively invariant signature for quadrics allows one to identify the type of CMS that caused linear selfcalibration to give ambiguous results. Finally, we explain that in the case of an artificial CMS, this signature allows us to uniquely recover the true self-calibration solution.

\section{Problem Formulation}

We consider the self-calibration problem for the case of a varying focal length but where the other intrinsic parameters are known. Let us review the classical formulation based on the Dual Absolute Quadric (DAQ) [22]. This is the planeenvelope of the Absolute Conic $\Omega_{\infty}$ (AC). Given a $3 \times 4$ perspective projection matrix $\mathrm{P}$, the image of the DAQ is given by the projection equation

$$
\omega^{*} \sim \mathrm{PQ}_{\infty}^{*} \mathrm{P}^{\top}
$$

where $\omega^{*}$ is the Dual Image of the Absolute Conic (DIAC).

We assume to be given a set of $n$ cameras obtained by means of projective reconstruction, that we write

$$
\mathbf{P}^{j}=\left(\begin{array}{c}
\mathbf{a}^{j \top} \\
\mathbf{b}^{j \top} \\
\mathbf{c}^{j \top}
\end{array}\right), \quad j=1, \ldots, n
$$

where $\mathbf{a}^{j}, \mathbf{b}^{j}$ and $\mathbf{c}^{j}$ are 4 -vectors representing planes.
Camera $j$ projects the DAQ to $\boldsymbol{\omega}^{* j}$. Under the above assumptions on the camera's intrinsic parameters, we may assume $\boldsymbol{\omega}^{* j} \sim \operatorname{diag}\left(\left(f^{j}\right)^{2},\left(f^{j}\right)^{2}, 1\right)$, where $f^{j}$ denotes the focal length of camera $j$. It can be readily seen that $Q_{\infty}^{*}$ then satisfies a set of four linear equations for each camera:

$$
\begin{aligned}
\mathbf{a}^{j \top} \mathbf{Q}_{\infty}^{*} \mathbf{a}^{j}-\mathbf{b}^{j \top} \mathbf{Q}_{\infty}^{*} \mathbf{b}^{j} & =0, & & \mathbf{a}^{j \top} \mathbf{Q}_{\infty}^{*} \mathbf{b}^{j}=0, \\
\mathbf{a}^{j \top} \mathbf{Q}_{\infty}^{*} \mathbf{c}^{j} & =0, & & \mathbf{b}^{j \top} \mathbf{Q}_{\infty}^{*} \mathbf{c}^{j}=0 .
\end{aligned}
$$

Solving those constraints for multiple cameras e.g., by optimizing in a linear least squares manner forms the basis of the dual linear self-calibration algorithms [11].

Additional constraints are that the DAQ $Q_{\infty}^{*}$ has rank 3 and is positive semi-definite. These constraints are nonlinear and are thus not taken into account in dual linear selfcalibration.

In this paper, we investigate critical motion sequences (CMS) for self-calibration, i.e., camera motions for which the problem does not have a unique solution. It is well known that there are CMSs that are generic for a given selfcalibration problem, in the sense that any algorithm will suffer from them, and that some algorithms may suffer from additional, artificial CMSs, due to not exploiting all available constraints [20]. In this paper, we study this issue for dual linear self-calibration. In section 3, we first review the generic CMSs and in section 4 we then derive all CMSs for dual linear self-calibration and explain which among these are artificial. After that, we show how articial CMSs can be detected from an ambiguous self-calibration result and that the ambiguity can indeed be resolved.

The definition of a CMS is as follows. Let the true cameras be $\mathrm{P}_{E}^{j} \sim \operatorname{diag}\left(f^{j}, f^{j}, 1\right) \mathrm{R}^{j}\left(\mathrm{I} \mid-\mathbf{t}^{j}\right)$. If there exists a $Q^{*}$ different from the true DAQ that satisfies all selfcalibration constraints (equations $(3,4)$ as well as the (spectral) rank-3 and positiveness constraints on $Q_{\infty}^{*}$ ), then the set of camera poses is termed a generic CMS. Artificial CMS for dual linear self-calibration are additional sets of camera poses for which there exists a $Q^{*}$ different from the true DAQ that only satisfies the linear equations $(3,4)$. In these cases, $Q^{*}$ is called a 'false $\mathbf{D} \boldsymbol{A} Q$ '.

\section{Generic Critical Motion Sequences}

The generic CMSs for self-calibration with varying focal length have been derived by Sturm [21] and Kahl et al. [10]. They proceeded as follows. They sought after virtual proper conics in 'regular' 3-space (in contrast to a search in 'dual' 3-space) other than the AC; if such a conic exists that satisfies all constraints, it is called a 'false' AC. With regard to their approach, the self-calibration equations $(3,4)$ have a simple meaning: they impose that the image of the $\mathrm{AC}$ is a circle, centered in the principal point. The image of a conic is such a circle, exactly if the cone, with centre the camera's optical centre and containing the conic, is a right (circular) 
cone. This geometric interpretation of the self-calibration constraints can now be used to reason on critical motions. Consider the existence of a false AC. Then, any camera position in a CMS, must satisfy the constraint that the cone, with centre the optical center and tangent to the false AC, is circular. Further, for any such camera position, the camera orientation must be such that the optical axis coincides with a revolution axis of that cone.

Based on this interpretation, Sturm and Kahl et al. derived all types of critical motions. This was done by considering all possible cases of false ACs and working out the camera positions and orientations according to the above definition. Basically Sturm and Kahl et al. (re)discovered (and proved) a specialised case of the following result: the locus of the vertices of a circular cone through a conic consists of its conjugate conics, which is illustrated in Fig. 1. By definition, two conjugate conics are conics lying on perpendicular planes and having an axis in common and the foci-pair (either both real or complex conjugate) of one conic must be coinciding with the intersection point-pair of the other with this axis [18, p245]. We wrote 'rediscovered' as this more general result has been known for a long time and is attributed to Dupin and Steiner in [19, p82].

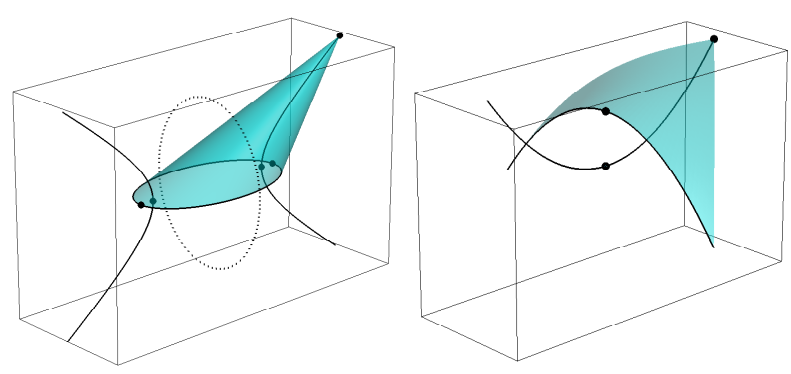

Figure 1. The two types of focal conic-sets: (left) ellipse / hyperbola / virtual-conic triplet; (right) parabola / parabola pair.

Focal conics have the remarkable general property of being conjugate (see text) and each conic completely determines the others.

\section{Artificial Critical Motion Sequences}

We now consider dual linear self-calibration i.e., the problem of estimating the DAQ without imposing that it is rank 3 and positive semi-definite i.e., without imposing that the $\mathrm{AC}$ is actually a conic and that it is a virtual conic. In other words, any quadric in dual form can now be a candidate false DAQ, under the condition that it satisfies the constraints expressed in $(3,4)$. As explained above, these convey exactly that the image of the DAQ is a circle centered in the image's principal point (to be precise, the dual of such a circle). A set of camera poses is thus critical if there exists another dual quadric that is projected to centered circles in all images. More exactly, the existence of such a CMS is equivalent to that of a $p$-parameter linear family of false DAQs with $p>0$. Terminology-wise, a CMS is said to be degenerate if the solution family is entirely degenerate i.e., if it consists of $\infty^{p}$ degenerate dual quadrics $\mathrm{D}^{*}$. It is not proved here but a condition for this is that $D^{*}$ is 'squashed' at infinity i.e., its points are located on the plane at infinity $\boldsymbol{\pi}_{\infty}$ i.e., $\mathrm{D}^{*} \boldsymbol{\pi}_{\infty}=\mathbf{0}_{4}$.

Consider now a dual quadric and a cone enveloping it whose vertex is the optical center. Its image is a centered circle (radii can be zero or imaginary) exactly if that cone is circular and if the camera's optical axis is aligned with a revolution axis of the cone. Based on this observation, CMS can be derived as in the works cited in the previous section, by considering all (Euclidean) types of dual quadrics, and determining the camera poses that satisfy the above constraints. This latter task is greatly simplified due to results in the projective geometry literature of the 19th- and the early 20 th-century $[15,18,19]$. In the following, we briefly summarize these and their implications for our study.

Before going further let us introduce the notions of (real and imaginary) foci and focal axes of a quadric (e.g., as in $[16, \mathrm{p} 339]$ or [18, p225]). Any point such that the tangentcone to a quadric with that point as vertex, is circular, is a focus of the quadric. Any line through a focus relative to which that cone is rotationally symmetric is a focal axis of the quadric. This is illustrated in Fig. 2. Note that for each focus, there is either a single focal axis, or all lines through it are focal axes (this is the case if the mentioned cone is isotropic i.e., contains the AC).

Regarding the issue of which dual quadrics other than the DAQ, get projected to a dual circle that is centred in the principal point, the answer is surprisingly enough quite simple: these are the quadrics for which the camera centre is a real focus and the optical axis a real focal axis. The result that we infer from this is fundamental; a sketch of the proof is given in the appendix.

Prop. 1 (Fundamental condition) $A$ motion sequence is critical iff there exists an irreducible ${ }^{1}$ quadric other than the DAQ such that:

1. any camera centre is a focus of the quadric,

2. any optical axis is a focal axis of the quadric.

In other words, it is equivalent to say that a camera motion is critical or that the camera moves on the 'focal curve' of some irreducible quadric while its optical axis, for each position, is a focal axis of the quadric through the camera centre. Note that, as a result, only the orientation of the camera's optical axis matters, whereas rotation about the optical axis is irrelevant.

What are the foci of a quadric? A first main classical result we use has been established long ago: a focus of a

\footnotetext{
${ }^{1} \mathrm{~A}$ (full) rank-4 or (deficient) rank-3 quadric in locus or envelope form.
} 


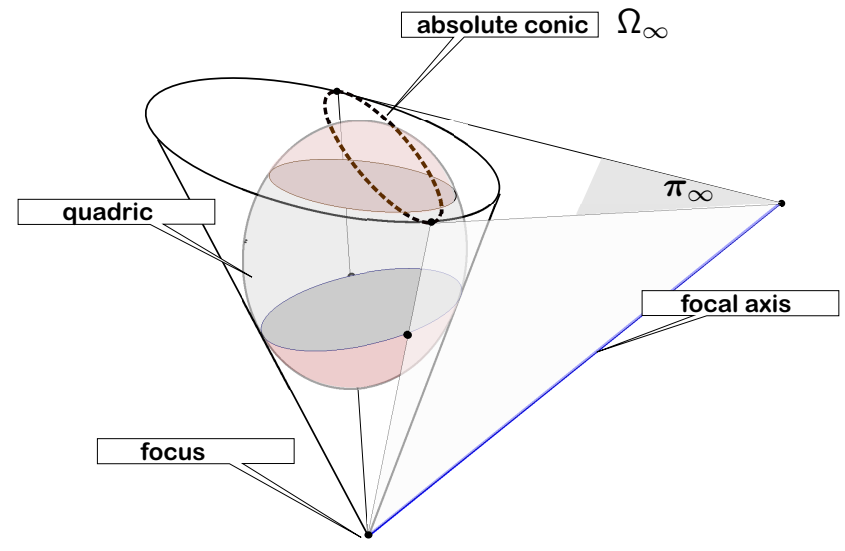

Figure 2. A focus and a focal axis of a quadric respectively coincide with the vertex and the revolution axis of a circular cone (i.e., having double contact with $\Omega_{\infty}$ ) enveloping the quadric (see text).

quadric is a point of its focal conics (a set of focal curves also called focals, $c f$. [19, p81]) which are conics lying on its principal planes (mutually orthogonal and such that any two of them share a principal axis). There are other properties, see e.g. [18]. Furthermore, it is known that there are only two types of focal conic-sets of a general quadric ${ }^{2}$ : (i) the parabola/parabola pair, (ii) the ellipse/hyperbola/virtual conic triplet (see Fig. 1). Other quadrics need special treatments that will be discussed in the sequel.

The second main result is due to Chasles (see [19, p81] and also [14]). It describes how to obtain the focal conics of a general quadric $Q$. Consider the confocal range of its dual $Q^{*}$, i.e. the $1 \mathrm{D}$ linear family of dual quadrics spanned by $Q^{*}$ and the DAQ $Q_{\infty}^{*}$. This family has in general four degenerate members; these, except $Q_{\infty}^{*}$, are exactly the envelopes of the focal conics of $Q$.

What are the focal axes of a quadric? A focal axis of a quadric is a line through which the two (complex) planes touching the AC are also tangent to the quadric [18, p224] (see Fig. 2). Thanks in particular to Plücker (see [19, p82]), it has been established that the assemblage of focal axes of a quadric consists of the generators i.e., the axes of pencils of planes, of the quadrics of its confocal range. The general notion of focal axis is less intuitive than that of focus and, as we are only interested in focal axes through foci, we will not look deeper into the theory of confocal quadrics.

\section{The Geometry of Criticality}

The above results, in particular Chasles' result, can be used to efficiently derive types of CMSs as shown in the following section. To determine all possible geometries of CMSs, it now suffices to compute the focal conics of quadrics of different Euclidean types and to describe the results

\footnotetext{
${ }^{2}$ Here, a general quadric is a proper quadric that is not of revolution.
}

in a generic geometric manner (this is done quite easily using a symbolic software like MAPLE). It is rather mechanical to carry out this procedure (see e.g., example 2 below), besides a few exceptions explained below (e.g., example 3). The results are summarized in table 1 , which contains all critical motions for the dual linear self-calibration problem.

To obtain these results, we needed to consider, besides the above classical geometric results, several other issues. First, these results only directly provide the camera positions in a CMS, the camera orientations are less directly given and are obtained algebraically. Second, as said previously, these results only hold for general proper quadrics; the cases of quadrics of revolution, spheres, and degenerate quadrics, need special treatment.

If we deal with a quadric of revolution, there is a major specificity: one conic in its focal-set is repeated and degenerates into either a rank-1 or a 'rank-0' conic (see Fig. 3). Note that no rank-2 focal conic does exist. The rank-1 focal conic is a ('repeated') line that coincides with the axis of revolution of the quadric. We will also call this a focal line. It contains two specialised foci called principal foci, which are the two foci of the conic obtained by meridiansection $^{3}$ of the quadric. In the special case of a sphere, this linear locus of foci further degenerates into a 'rank-0 conic', meaning that every point in 3-space is a focus of the sphere. The sphere has a single principal focus, its centre. Note that all lines passing through a principal focus, are focal axes of the quadric.

Let us consider, for these special cases, the nature of focal axes that pass through a focus (keep in mind that they determine the orientation of optical axes in CMSs). Consider first the general case of a quadric having general focal conics. For any point on such a focal conic, the focal axis passing through it coincides with the tangent line to that focal conic at that point which is coplanar with the focal conic. Next, we consider the case of a focal line, which happens for quadrics of revolution, see above. For any point on a focal line, the focal axis passing through that point is the focal line itself (which is always a real line). An exception are the two principal foci, where any line passing through them is a focal axis. Finally, in the case of a sphere, every point in 3 -space is a focus, as explained above, and through it there is just one focal axis which is the line joining that point and the centre of the sphere. Again, an exception is the centre of the sphere itself, for which all lines passing through it, are focal axes (see Fig. 3).

There are two classes of confocal ranges for which the locus of foci is the entire 3-space i.e., a focal space. The first includes $(i)$ all confocal (i.e., concentric) ranges of spheres (type (R4) in table 1) and the second (ii) all degenerate confocal ranges, for which arbitrary values of $\alpha_{1}, \alpha_{2}$ satisfy $\operatorname{det}\left(\alpha_{1} \mathrm{Q}^{*}+\alpha_{2} \mathrm{Q}_{\infty}^{*}\right)=0$ (type (D)). Here, degenerate con-

\footnotetext{
${ }^{3}$ Any planar section of the quadric through its axis of revolution.
} 
focal quadric ranges correspond to ranges of conics at infinity with the dual absolute conic $\Omega_{\infty}^{*}$ as member (cf. $\left.\S 4\right)$. What distinguishes $(i)$ and (ii) is that, in the former case, the focal axes through foci concur at a finite (unique) principal focus; in the latter case, they concur at a principal foci at infinity and hence all focal axes are parallel.

We now give two examples carrying some technical details on how to determine the real points of the focal conics. Other cases are solved in very similar ways.

Example 2 We here determine the foci and focal axes of a central quadric $\mathrm{Q}$ (class G1). Write the envelope of $\mathrm{Q}$ in Euclidean canonical form as

$$
\mathrm{Q}^{*}=\operatorname{diag}\left(a_{1}, a_{2}, a_{3},-1\right)
$$

The parameters of the degenerate envelopes of the confocal range determined by $\mathrm{Q}^{*}$ are the generalized eigenvalues of the matrix-pair $\left(Q^{*}, Q_{\infty}^{*}\right)$ which can be easily computed since $Q_{\infty}^{*}=\operatorname{diag}(1,1,1,0)$. These parameters are $a_{1}, a_{2}, a_{3}$ and $\infty$ (the latter is the parameter of $\mathrm{Q}_{\infty}^{*}$ ). Hence it is straightforward to compute, in this order, the matrices of the degenerate envelopes -other than $\mathrm{Q}_{\infty}^{*}$ - and their (ordinary) eigenvalues:

$$
\begin{aligned}
& \mathbf{F}_{1}^{*}=\left(\begin{array}{cccc}
0 & 0 & 0 & 0 \\
0 & a_{2}-a_{1} & 0 & 0 \\
0 & 0 & a_{3}-a_{1} & 0 \\
0 & 0 & 0 & -1
\end{array}\right) ; \operatorname{eig}\left(\mathrm{F}_{1}^{*}\right)=\left(\begin{array}{c}
0 \\
-1 \\
a_{3}-a_{1} \\
a_{2}-a_{1}
\end{array}\right) \\
& \mathbf{F}_{2}^{*}=\left(\begin{array}{cccc}
a_{1}-a_{2} & 0 & 0 & 0 \\
0 & 0 & 0 & 0 \\
0 & 0 & a_{3}-a_{2} & 0 \\
0 \\
-1 \\
a_{3}-a_{2} \\
a_{1}-a_{2}
\end{array}\right) ; \operatorname{eig}\left(\mathrm{F}_{2}^{*}\right)=\left(\begin{array}{c}
0 \\
-1 \\
a_{2}-a_{3} \\
a_{1}-a_{3}
\end{array}\right) ; \operatorname{eig}\left(\mathrm{F}_{3}^{*}\right)=\left(\begin{array}{cccc}
a_{1}-a_{3} & 0 & 0 & 0 \\
0 & a_{2}-a_{3} & 0 & 0 \\
0 & 0 & 0 & 0 \\
0 & 0 & 0 & -1
\end{array}\right)
\end{aligned}
$$

The $\mathrm{F}_{k}^{*}$ 's are matrices of three rank-3 dual quadrics encoding three focal conics on the principal planes of the quadric (point-equations of these focals can be easily derived). Assume $\mathrm{Q}$ to be general e.g., $a_{1}<a_{2}<a_{3}$ and $a_{1} a_{2} a_{3} \neq 0$. The first two point-equations represent conjugate focal conics i.e., a (real) ellipse on the YZ-plane and a (real) hyperbola on the $X Z$-plane respectively, while the third one represents a virtual ellipse on the $X Y$-plane. Each tangent line to the conic on the supporting plane of the conic is the axis of a pencil of planes of $\mathrm{F}_{k}^{*}$ and thus is a focal axis of $\mathrm{Q}$.

Example 3 We now determine the foci and focal axes of an elliptic paraboloid of revolution $\mathrm{Q}(\mathrm{R} 3)$. Write the envelope of $\mathrm{Q}$ in Euclidean canonical form as

$$
\mathrm{Q}^{*}=\left(\begin{array}{cccc}
1 & 0 & 0 & 0 \\
0 & 1 & 0 & 0 \\
0 & 0 & 0 & 2 / b \\
0 & 0 & 2 / b & 0
\end{array}\right) \quad b \neq 0
$$

The parameters of the degenerate envelopes of the confocal range of $\mathrm{Q}^{*}$ are the generalized eigenvalues of $\left(\mathrm{Q}^{*}, \mathrm{Q}_{\infty}^{*}\right)$. One gets generalized eigenvalues equal to 1 and $\infty$, both with multiplicity two ( $\infty$ is the parameter of $\mathrm{Q}_{\infty}^{*}$ ). The matrix of the single 'focal' degenerate envelope (with parameter 1) is given below with its (ordinary) eigenvalues:

$$
F^{*}=\left(\begin{array}{cccc}
0 & 0 & 0 & 0 \\
0 & 0 & 0 & 0 \\
0 & 0 & -1 & 2 / b \\
0 & 0 & 2 / b & 0
\end{array}\right) ; \quad \operatorname{eig}\left(F^{*}\right)=\frac{1}{2 b}\left(\begin{array}{c}
0 \\
0 \\
-b+\left(b^{2}+16\right)^{1 / 2} \\
-b-\left(b^{2}+16\right)^{1 / 2}
\end{array}\right)
$$

As the product of the $3 r d$ and 4 th eigenvalues yields $-4 / b^{2}$, one deduces that $\mathrm{F}^{*}$ is a real point-pair, say $\mathrm{F}^{*}=\mathbf{f g}^{\top}+\mathbf{g f}^{\top}$ where $\mathbf{f}, \mathbf{g} \in \mathbb{R}^{4}$, formed by the two principal foci of the quadric. By inspecting the element $F_{44}^{*}=0=2 f_{4} g_{4}$, one can see that $\mathbf{f}$ or $\mathbf{g}$-but not both-is at infinity. The line spanned by $\mathbf{f}$ and $\mathbf{g}$ is a focal line i.e., a rank-1 focal conic. Through each of $\mathbf{f}$ and $\mathbf{g}$, there passes $\infty^{2}$ planes (a star of planes) and hence $\infty^{2}$ lines which are the focal axes of $Q$, including the line through $\mathbf{f}$ and $\mathbf{g}$.

Along these lines, we can derive all CMSs ; they are summarized in table 1. Naturally, they subsume the generic critical motions summarized in section 3. Concretely, the classes of motions (G1), (R1) and (D) are generic critical motions, all others are artificial ones for the dual linear selfcalibration approach (see Fig. 4).
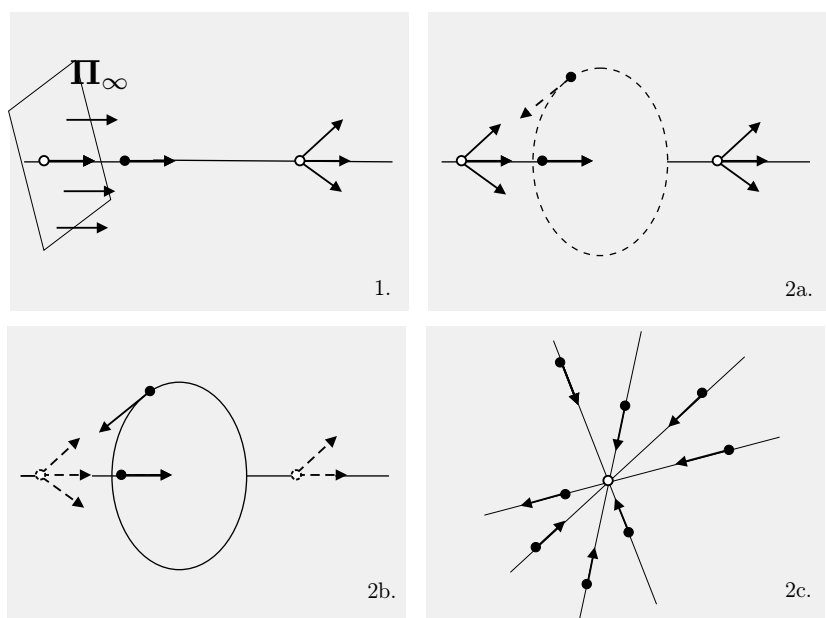

Figure 3. Four degenerate types of focus loci (black dots), including principal foci (white dots), and assemblage of focal axes through them (arrows). The dashed style indicates virtual objects. The general case is shown in Fig. 1.

\section{How to Detect Artificial Critical Motions}

In this section, we show how one may detect and handle artificial CMSs, starting from a projective reconstruction. 


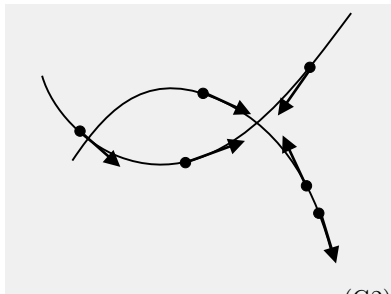

(G2)

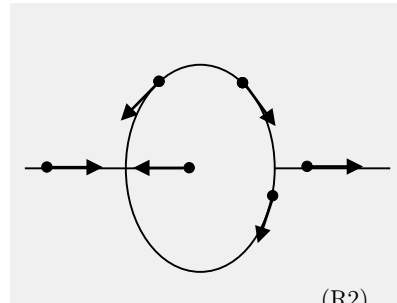

(R2)

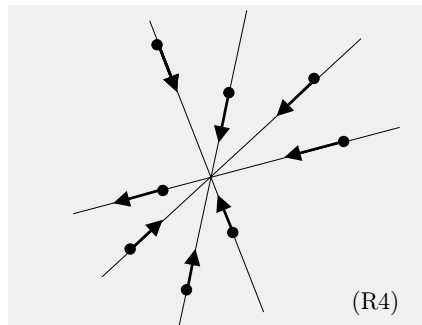

Figure 4. The three classes (G2), (R2) and (R4) of artificial CMS completing the known generic CMS. The ambiguity for the DAQ is $1 \mathrm{D}$ if the CMS is rank-2 i.e., it includes at least three cameras with at least one on the circle for (R2).

\begin{tabular}{|c|c|c|}
\hline Quadric Confocal Ranges & $\begin{array}{l}\text { Degenerate envelopes (focal conics) } \\
\text { and associated signature sequence }\end{array}$ & $\begin{array}{l}\text { Critical motions: locus } \mathscr{L} \text { of camera centres } \\
\text { and associated assemblage } \mathscr{A} \text { of optical axes. }\end{array}$ \\
\hline $\begin{array}{l}\text { (G1) General virtual ellipsoids, el- } \\
\text { lipsoids and hyperboloids of one or } \\
\text { two sheets }\end{array}$ & $\begin{array}{c}\left\{Q_{\infty}^{*}, Q_{\text {virt }}^{*}, E^{*}, H^{*}\right\} \\
\{(3,0),(3,0),(2,1),(2,1)\}\end{array}$ & $\begin{array}{l}\mathscr{L} \text { is a conjugate conic-pair formed by a general } \\
\text { ellipse and a general hyperbola; } \mathscr{A} \text { consists of axes } \\
\text { tangent to these conics in their supporting planes. }\end{array}$ \\
\hline $\begin{array}{l}\text { (G2) General elliptic and hyper- } \\
\text { bolic paraboloids }\end{array}$ & $\begin{array}{c}\left\{\mathrm{Q}_{\infty}^{*} \times 2, \mathrm{P}_{1}^{*}, \mathrm{P}_{2}^{*}\right\} \\
\{((3,0)),(2,1),(2,1)\}\end{array}$ & $\begin{array}{l}\mathscr{L} \text { is a conjugate conic-pair formed by two general } \\
\text { parabolas; } \mathscr{A} \text { consists of axes tangent to these con- } \\
\text { ics in their supporting planes. }\end{array}$ \\
\hline $\begin{array}{l}\text { (R1) Prolate ellipsoids and hyper- } \\
\text { boloids of two sheets, oblate vir- } \\
\text { tual ellipsoids }\end{array}$ & $\begin{array}{c}\left\{\mathrm{Q}_{\infty}^{*}, \mathrm{C}_{\mathrm{virt}}^{*},\left(\mathbf{F} \mathbf{F}^{\prime \top}+\mathbf{F}^{\prime} \mathbf{F}^{\top}\right) \times 2\right\} \\
\{(3,0),(3,0),((1,1))\}\end{array}$ & $\begin{array}{l}\mathscr{L} \text { is a single line; } \mathscr{A} \text { consists of } \mathscr{L} \text { and two stars } \\
\text { of axes through two fixed finite points on } \mathscr{L} .\end{array}$ \\
\hline $\begin{array}{l}\text { (R2) oblate ellipsoids and hyper- } \\
\text { boloids of one sheet; prolate vir- } \\
\text { tual ellipsoids }\end{array}$ & $\begin{array}{c}\left\{\mathrm{Q}_{\infty}^{*}, \mathrm{C}^{*},\left(\mathbf{F}_{+} \mathbf{F}_{-}^{\top}+\mathbf{F}_{-} \mathbf{F}_{+}^{\top}\right) \times 2\right\} \\
\{(3,0),(2,1),((2,0))\}\end{array}$ & $\begin{array}{l}\mathscr{L} \text { consists of a circle and a line which cuts ortho- } \\
\text { gonally its centre; } \mathscr{A} \text { consists of this line and the } \\
\text { axes tangent to the circle in its supporting plane. }\end{array}$ \\
\hline $\begin{array}{l}\text { (R3) Elliptic paraboloids of re- } \\
\text { volution }\end{array}$ & $\begin{array}{c}\left\{\mathrm{Q}_{\infty}^{*} \times 2,\left(\mathbf{F F}_{\infty}^{\prime \top}+\mathbf{F}_{\infty}^{\prime} \mathbf{F}^{\top}\right) \times 2\right\} \\
\{((3,0)),((1,1))\}\end{array}$ & $\begin{array}{l}\mathscr{L} \text { is a single line; } \mathscr{A} \text { consists of two stars of axes } \\
\text { through two fixed points on } \mathscr{L} \text {, one finite and the } \\
\text { other infinite. }\end{array}$ \\
\hline (R4) Spheres & $\begin{array}{l}\left\{\mathbf{Q}_{\infty}^{*}, \mathbf{C C}^{\top} \times 3\right\} \\
\{(3,0),(((1,0)))\}\end{array}$ & $\begin{array}{l}\mathscr{L} \text { is the } 3 \text {-space; } \mathscr{A} \text { consists of a star of nonparallel } \\
\text { axes, through a fixed finite point. }\end{array}$ \\
\hline (D) Degenerate confocal ranges & $\infty^{1}$ degenerate envelopes & $\mathscr{L}$ is the 3 -space; $\mathscr{A}$ consists of all parallel axes. \\
\hline
\end{tabular}

Table 1. All types of critical motions, induced by a seven-class partition of confocal ranges w.r.t. signature sequences. Glossary: virtual degenerate envelopes are marked by 'virt' - objects at infinity are marked by ' $\infty$ '. $\mathrm{E}_{\text {virt }}^{*}$ : virtual ellipse, $\mathrm{E}^{*}$ : ellipse, $\mathrm{H}^{*}$ : hyperbola, $\mathrm{P}_{j}^{*}$ : parabola $j, C^{*}$ : circle, $\left(\mathbf{F}, \mathbf{F}^{\prime}\right)$ : (real) principal foci-pair, $\left(\mathbf{F}_{+}, \mathbf{F}_{-}\right)$: (complex conjugate) principal foci-pair, $\mathbf{C}$ : single principal focus.

This is based on the notion of signature of a degenerate dual quadric $D^{*}$.

Let $\rho$ and $\nu$ be respectively the numbers of positive and negative eigenvalues of $D^{*}$. The signature of $D^{*}$ is the pair $\left(\xi_{1}, \xi_{2}\right)$ with $\xi_{1} \equiv \max (\rho, \nu)$ and $\xi_{2} \equiv \min (\rho, \nu)$ while $\xi_{1}+\xi_{2}=$ rank $D^{*}$. A crucial property of signature and rank is that they are invariant to homographies [8, p74].

A projective description of a confocal range, spanned by
$\mathrm{Q}_{1}^{*}$ and $\mathrm{Q}_{2}^{*}$, is now given by its signature sequence

$$
\left\{\left(\cdots\left(\xi_{1}^{1}, \xi_{2}^{1}\right) \cdots\right), \ldots,\left(\cdots\left(\xi_{1}^{r}, \xi_{2}^{r}\right) \cdots\right)\right\}
$$

where $r \in\{1 . .4\}$ and

- $\left(\xi_{1}^{r}, \xi_{2}^{r}\right)$ is the signature of any degenerate $\mathrm{Q}_{1}^{*}-\lambda_{r} \mathrm{Q}_{2}^{*}$, where $\lambda_{r}$ is a generalized eigenvalue of $\left(Q_{1}^{*}, Q_{2}^{*}\right)$;

- the number of brackets around a signature $(c f$. 2nd column of table 1) indicates the number of times that $Q_{1}^{*}-\lambda_{r} Q_{2}^{*}$ is repeated in the set of degenerate envel- 
opes i.e., the algebraic eigenvalue multiplicity of $\lambda_{r}$.

The signature sequence describes the four (possibly repeated) degenerate dual quadrics of the range. It yields a projective description as it relies on signatures and multiplicities of these dual quadrics which are the same in any projective representation ${ }^{4}$.

The signature sequences including the signatures of the false DAQs for all cases of CMSs, are given in table 1. They are at the basis of identifying and handling artificial CMSs, as described in the following.

Let us consider the solution of the linear self-calibration equations $(3,4)$. Of course, if there are ambiguous solutions, we are in the presence of a critical motion. Since we deal with linear equations, ambiguous solutions correspond to a linear family of dual quadrics. We assume that there is only a $1 \mathrm{D}$ linear family. This is the most practical common case as soon as there are $n \geq 3$ critical cameras taken anywhere in the critical sequence ${ }^{5}$, except for (R2) in which at least one must be on the circle, cf. Fig. 4 . This can be easily proved e.g., using MAPLE. We first compute the four degenerate members of that linear family, e.g. by computing the generalized eigenvalues of a pair of dual quadrics that span the family. Then, for each of these degenerate dual quadrics, we compute its signature. A first observation is that the signature sequence allows one to uniquely identify the class of CMSs ( $c f$. 2nd column of table 1). A second observation is that all false DAQs of all artificial critical motions (classes (G2), (R2), (R3) and (R4)) have a signature that is different from that of the true DAQ, $(3,0)$. Hence, in the presence of an artificial CMSs, the true DAQ can be identified without ambiguity, as the single one having signature $(3,0)$. In conclusion, artificial CMSs can, when taking into account all self-calibration constraints after solving the linear equation system, always be disambiguated. Figure 5 shows a typical example where our algorithm resolves an artificial CMS.

\section{Conclusions}

Our theoretical results have two major consequences in practical applications. First, existing SfM implementations using dual linear self-calibration $[11,12,17]$ are known to be unstable due to degeneracies and noise. But degeneracies are intrinsically due to critical motions. Knowing the artificial CMSs will allow one to avoid them. Second, encapsulating the spectral constraints in the signature sequence, we proved that those are discriminant enough to find the true DAQ within a 1D family of potential DAQs. In other words, we stated that the spectral constraints can be safely enforced a posteriori, making it possible to avoid ambiguous self-calibration in the presence of artificial CMSs (refer

\footnotetext{
${ }^{4}$ A signature is projectively invariant by Sylvester's law of inertia. The multiplicity of a generalized eigenvalue also has this property.

${ }^{5}$ We do not consider the class (D) here.
}

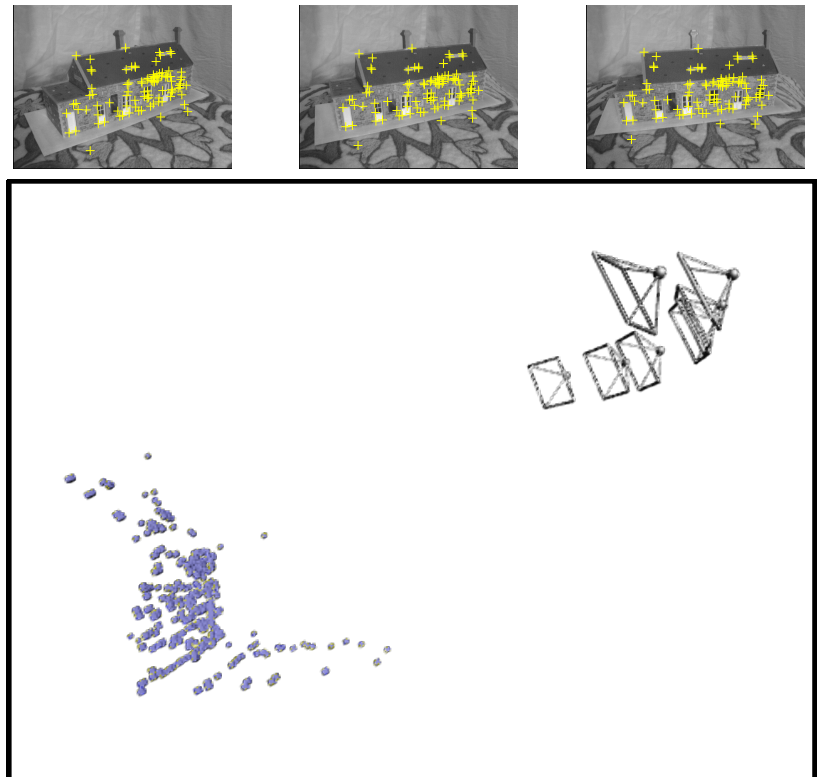

Figure 5. (3D interactive graphic) We ran the dual self-calibration linear algorithm from the publicly available "Model House" image sequence (www.robots.ox.ac.uk/vgg/data). To get the projective cameras, we used Sturm-Triggs' projective factorization (see [8, p444]), followed by projective bundle adjustment. Only the first six (out of seven) frames were considered, forming a subsequence in which 94 2D points were matched all over. Regarding the selfcalibration solution given by a SVD-based algorithm (similar to A5.3 in [8, p592]), after computing the ratios of the first singular value to the last three ones, respectively equal to $5.70 e+004$, $9.43 e+002$ and 32.83 , we concluded that we were facing a critical motion, which was confirmed by visualising the 'meaningless' $3 \mathrm{D}$ metric reconstruction associated with the lowest singular value. Hence, the solution for the DAQ is included in a tangential pencil of quadrics $\alpha_{1} \mathrm{Q}_{1}^{*}+\alpha_{2} \mathrm{Q}_{2}^{*}$. We normalise the $\mathrm{Q}_{i}^{*}$ 's by dividing them by their largest singular values, and next assume that, regarding the signatures of the degenerate quadrics $Q_{1}^{*}-\lambda_{r} Q_{2}^{*}$ where the $\lambda_{r}$ 's are the generalized eigenvalues of $\left(\mathrm{Q}_{1}^{*}, \mathrm{Q}_{2}^{*}\right)$, any value lower than $10 e-3$ is zero. The obtained signature sequence (with no other 'numerical trick') was $\{(3,0),(((1,0)))\}$. This indicates that all the cameras fixates a point, whose matrix is associated with the signature corresponding to $(1,0)$. We picked up as solution the degenerate envelope associated with $(3,0)$ and obtained the reconstruction of points and cameras which is displayed here, completed by reconstructions of additional pair-wise matched points.

to the interactive graphic in Fig. 5).

\section{References}

[1] A. Bartoli and P. Sturm. The 3D line motion matrix and alignment of line reconstructions. IJCV, 57(3):159-178, 2004.

[2] B. Bocquillon, A. Bartoli, P. Gurdjos, and A. Crouzil. On constant focal length self-calibration from multiple views. In CVPR, 2007. 
[3] M. Chandraker, S. Agarwal, D. Kriegman, and S. Belongie. Globally optimal affine and metric upgrades in stratified autocalibration. In ICCV, 2007.

[4] O. Faugeras. What can be seen in three dimensions with an uncalibrated stereo rig? In ECCV, 1992.

[5] O. Faugeras, Q. Luong, and T. Papadopoulou. The geometry of multiple images. MIT Press, 2001.

[6] S. Finsterwalder. Die geometrischen Grundlagen der Photogrammetrie. Jahresbericht Deutscher Mathematik, 6:1-44, 1899.

[7] R. Hartley, R. Gupta, and T. Chang. Stereo from uncalibrated cameras. In CVPR, pages 761-764, 1992.

[8] R. Hartley and A. Zisserman. Multiple view geometry in computer vision. Cambridge Univ. Press, 2003.

[9] A. Heyden and K. Åström. Minimal conditions on intrinsic parameters for Euclidean reconstruction. In ACCV, 1997.

[10] F. Kahl, B. Triggs, and K. Åström. Critical motions for autocalibration when some intrinsic parameters can vary. JMIV, 13(2):131-146, 2000.

[11] M. Pollefeys. Self-calibration and metric 3D reconstruction from uncalibrated image sequences. Ph.D. Thesis, ESATPSI, K.U.Leuven, 1999.

[12] M. Pollefeys, R. Koch, and L. Van Gool. Self-calibration and metric reconstruction inspite of varying and unknown intrinsic camera parameters. IJCV, 32(1):7-25, 1999.

[13] J. Ponce, K. McHenry, T. Papadopoulo, M. Teillaud, and B. Triggs. On the absolute quadratic complex and its application to autocalibration. In CVPR, volume 1, 2005.

[14] E. Primrose. Foci of a quadric. The Mathematical Gazette, 40(332):135-136, may 1956.

[15] G. Salmon. A treatise on the analytic geometry of three dimensions, volume 1 of 4 th edition. Dublin, Hodges, Figgis, \& Co, 1882.

[16] J. Semple and G. Kneebone. Algebraic projective geometry. Oxford University Press, 1952.

[17] Y. Seo and A. Heyden. Auto-calibration by linear iteration using the DAC equation. IVC, 22(11):919 - 926, 2004.

[18] D. Sommerville. Analytical geometry of three dimensions. Cambridge University Press, 1939.

[19] O. Staude and A. Grévy. Géométrie algébrique dans l'espace. In J. Molk, editor, Encyclopédie des Sciences Mathématiques Pures et Appliquées, volume III, chapter 4, pages 1-164. Jacques Gaby, 1992 edition, 1904-1916.

[20] P. Sturm. A case against Kruppa's equations for camera selfcalibration. PAMI, 22(10):1199-1204, september 2000.

[21] P. Sturm. Critical motion sequences for the self-calibration of cameras and stereo systems with variable focal length. IVC, 20(5-6):415-426, march 2002.

[22] B. Triggs. Autocalibration and the absolute quadric. In CVPR, 1997.

[23] A. Valdés and J. Ignacio Ronda. Camera autocalibration and the calibration pencil. JMIV, 23(2):167-174, 2005.

\section{Appendix}

Due to lack of space, only a sketch of the proof of proposition 1 can be given here. Before, we remind some known projective facts $[15,16,18]$. Except for the plane at infinity $\boldsymbol{\pi}_{\infty}$, which is real, all the planes of the DAQ $Q_{\infty}^{*}$ are complex conjugate ; hence all the tangent-lines ${ }^{6}$ of $Q_{\infty}^{*}$ are also complex conjugate. We refer to such pairs as isotropic plane-pairs and isotropic line-pairs respectively. An important property of an isotropic plane-pair is to be invariant under rotations around its axis i.e., the intersection line of its two planes.

We also require an alternate definition of the focus of quadric, which can be found in [15, p127] and actually applies in dual 3-space for an algebraic surface of any order. 'A focus is a point through which can be drawn two lines, each touching the surface and meeting the absolute conic, and such that the tangent plane to the surface through either also touches the absolute conic.'

Now, let us reveal the complex geometry of equations $(3,4)$ by considering the algebraically equivalent pair:

$$
\begin{aligned}
\left(\mathbf{a}^{j} \pm i \mathbf{b}^{j}\right)^{\top} \mathbf{Q}_{\infty}^{*}\left(\mathbf{a}^{j} \pm i \mathbf{b}^{j}\right) & =0, \\
\mathbf{c}^{j \top} \mathbf{Q}_{\infty}^{*}\left(\mathbf{a}^{j} \pm i \mathbf{b}^{j}\right) & =0,
\end{aligned}
$$

with $i^{2}=-1$. Thus, we treat $\mathbf{a}^{j}$ (resp. $\mathbf{b}^{j}$ ) in (2) as the real (resp. imaginary) part of a complex conjugate plane-pair. Since any camera $\mathrm{P}^{j}, c f$. (2), satisfies equations $(7,8)$, then (i) $\boldsymbol{\pi}_{ \pm}^{j}=\mathbf{a}^{j} \pm i \mathbf{b}^{j}$ is an isotropic plane-pair whose axis is the optical axis, (ii) the intersection of $\boldsymbol{\pi}_{ \pm}^{j}$ and $\mathbf{c}^{j}$, denoted $\boldsymbol{\pi}_{ \pm}^{j} \curlywedge \mathbf{c}^{j}$, is an isotropic line-pair through the optical centre $\mathbf{O}^{j}$. Indeed, using (7), (i) holds by definition of an isotropic plane-pair. Using $(7,8)$, (ii) also holds by definition as the intersection of two planes $\mathbf{p}$ and $\mathbf{q}$ is a tangent line of a quadric envelope $X^{*}$ iff both equations ${ }^{7} \mathbf{p}^{\top} \mathbf{X}^{*} \mathbf{p}=0$ and $\mathbf{p}^{\top} \mathbf{X}^{*} \mathbf{q}=0$ are satisfied [15, pp147-148].

That said the proof can be sketched: (i) A camera $\mathrm{P}^{j}$ of the form (2) is critical w.r.t. $\mathrm{X}^{*} ¥ \mathrm{Q}_{\infty}^{*}$ iff (ii) $\mathrm{P}^{j}$ and $\mathrm{X}^{*}$ satisfy $(7,8)$. Using a similar reasoning as above, an equivalent condition is that (iii) the isotropic plane-pair $\boldsymbol{\pi}_{ \pm}$is also a plane-pair of $\mathrm{X}^{*}$ and (iv) the isotropic line-pair $\boldsymbol{\pi}_{ \pm}^{j} \curlywedge \mathbf{c}^{j}$ is also a tangent line-pair ${ }^{6}$ of $X^{*}$. When this holds, the optical centre is a point through which there pass two isotropic lines tangent to $X$ and such that the isotropic planes through the lines belong to $X^{*}$. By the alternate definition, the optical axis is a real focal axis of $X$ and the optical centre is a real focus on the focal axis.

\footnotetext{
${ }^{6}$ What is the tangent line to a dual quadric? A tangent line to a quadric locus is a line which meets the quadric at two coinciding points. Through a line there are just two planes which belong to a dual quadric; this line is a tangent line to the dual quadric if the two planes coincide.

${ }^{7} \mathbf{p}$ should be a plane of $X^{*}$ while $\mathbf{q}$ should not.
} 